\title{
Ehrenfest Approach to the Adiabatic Invariants and Calculation of the Intervals of Time Entering the Energy Emission Process in Simple Quantum Systems
}

\author{
Stanisław Olszewski \\ Institute of Physical Chemistry, Polish Academy of Sciences, Warsaw, Poland \\ Email: olsz@ichf.edu.pl
}

How to cite this paper: Olszewski, S (2020) Ehrenfest Approach to the Adiabatic Invariants and Calculation of the Intervals of Time Entering the Energy Emission Process in Simple Quantum Systems. Journal of Quantum Information Science, 10, 1-9.

https://doi.org/10.4236/jqis.2020.101001

Received: December 19, 2019

Accepted: March 28, 2020

Published: March 31, 2020

Copyright $\odot 2020$ by author(s) and Scientific Research Publishing Inc. This work is licensed under the Creative Commons Attribution International License (CC BY 4.0).

http://creativecommons.org/licenses/by/4.0/

\begin{abstract}
In the first step, the Ehrenfest reasoning concerning the adiabatic invariance of the angular orbital momentum is applied to the electron motion in the hydrogen atom. It is demonstrated that the time of the energy emission from the quantum level $n+1$ to level $n$ can be deduced from the orbital angular momentum examined in the hydrogen atom. This time is found precisely equal to the time interval dictated by the Joule-Lenz law governing the electron transition between the levels $n+1$ and $n$. In the next step, the mechanical parameters entering the quantum systems are applied in calculating the time intervals characteristic for the electron transitions. This concerns the neighbouring energy levels in the hydrogen atom as well as the Landau levels in the electron gas submitted to the action of a constant magnetic field.
\end{abstract}

\section{Keywords}

Aadiabatic Invariants, Time of the Energy Emission Process Referred to the Mechanical Parameters of a Quantum System, Hydrogen Atom, The Landau Levels in the Electron Gas

\section{Introduction}

In spite of its evident importance, the time parameter-for various reasons-is not much welcomed by the quantum physicists. Instead of its direct calculation, a probabilistic approach to the electron transitions has been developed from the very beginning of the quantum theory [1] [2] [3] [4] [5].

The aim of the present paper is to examine the time intervals characteristic for 
the quantum emission process, partly on the basis of the Ehrenfest treatment of the adiabatic invariants [6] [7] and partly with the aid of a study of the mechanical properties of electrons entering the simple quantum systems.

A non-probabilistic approach to the calculation of the emission time of electron energy was based on a suitable modification of the classical Joule-Lenz law [8]. For the emission concerning the change of the electron energy due to transition between two neighbouring quantum levels,

$$
n+1>n,
$$

so

$$
E_{n+1}>E_{n},
$$

and the emitted energy was

$$
\Delta E=E_{n+1}-E_{n} .
$$

The classical Joule-Lenz law adapted to the quantum emission process [9] [10] [11] gave for the emission time $\Delta t$ the formula

$$
\Delta E \Delta t=h,
$$

so

$$
\Delta t=\frac{h}{\Delta E}
$$

where $\Delta E$ is the expression given in (3).

The first aim of the present paper is to point out that relation exactly equivalent to that given in (5) can be obtained on the basis of the Ehrenfest reasoning concerning the invariant of the electron angular momentum entering the hydrogen atom [6] [7]. But another step worth to note is that $\Delta t$ obtained from the relation (5) can be approached also by examining the mechanical parameters of the electron particle in a quantum system. This observation-made first for the hydrogen atom [12] and repeated in the present paper in order to remove a printing error done in [12] - is demonstrated to hold also for the case of the Landau energy levels obtained in the electron gas in effect of the action of a constant magnetic field; see Secs. 6 and 7.

\section{The Orbital Angular Momentum of the Electron being an Adiabatic Invariant in the Hydrogen Atom}

The electron orbital angular momentum in the hydrogen atom is given by the formula

$$
p_{\varphi}=m r^{2} \dot{\varphi} .
$$

In (6) the $r$ is a distance between the electron of mass $m$ and position of the atomic nucleus, the $\dot{\varphi}$ is the speed of change of the angle $\varphi$ between the direction of the electron observed from the nucleus and some constant direction.

Ehrenfest identifies the frequency $v$ of the electron circulation about a fixed 
atomic nucleus at a distance $r$ with the formula

$$
v= \pm \frac{\dot{q}}{2 \pi}
$$

The electron rotates uniformly, uninfluenced by any supplementary force excepting the electrostatic attraction force. The adiabatic invariant is

$$
\frac{2 \bar{E}_{\mathrm{kin}}}{v}=\iint \mathrm{d} q \mathrm{~d} p
$$

where $\bar{E}_{\text {kin }}$ is the average kinetic energy of a particle. The invariance means that the right-hand side of (8) should retain its original values

$$
0, h, 2 h, 3 h, \cdots
$$

Because of (7) and in virtue of the relation

$$
2 \bar{E}_{\mathrm{kin}}=2 E_{\mathrm{kin}}=p \dot{q}
$$

We expect that the variable $p$ entering (8) and (10) should take the values

$$
p=0, \pm \frac{h}{2 \pi}, \pm \frac{2 h}{2 \pi}, \cdots
$$

\section{Quantum Formulae for Systems Representing the Periodic Motion about a Centre of Attraction of the Newtonian Kind}

If $\chi$ is a potential representing a central attractive force, the differential equations satisfied in the motion plane expressed in the polar coordinates $(r, \varphi)$ are:

$$
m \ddot{r}-m r \dot{\varphi}^{2}+\frac{\mathrm{d} \chi}{\mathrm{d} r}=0
$$

and

$$
\frac{\mathrm{d}}{\mathrm{d} t}\left(m r^{2} \dot{\varphi}\right)=0
$$

where $t$ is the time variable. The integration of Equation (13) gives readily an adiabatic invariant equivalent to a constant angular momentum $p=p_{\varphi}$ represented in (6).

In the next step let $\chi$ be an attractive electrostatic potential between the nucleus of charge $\mathrm{Ze}$ and the electron:

$$
\chi=-\frac{Z e^{2}}{r}=-\frac{e^{2}}{r}
$$

on condition in the last step in (14) we put

$$
Z=1 \text {. }
$$

In effect we obtain from (6) and (12):

$$
m \ddot{r}=m r \dot{\varphi}^{2}-\frac{\mathrm{d} \chi}{\mathrm{d} r}=m r\left(\frac{p_{\varphi}}{m r^{2}}\right)^{2}-\frac{e^{2}}{r^{2}}=p_{\varphi}^{2} \frac{1}{m r^{3}}-\frac{e^{2}}{r^{2}} .
$$


In the hydrogen atom, the constant radius $r$ of the electron orbit implies

$$
\ddot{r}=0 .
$$

Therefore (16) is reduced to the equation

$$
\frac{p_{\varphi}^{2}}{m r^{3}}-\frac{e^{2}}{r^{2}}=0
$$

which gives

$$
\frac{p_{\varphi}^{2}}{m r}=e^{2} .
$$

By assuming a quantized angular momentum $p_{\varphi}$ in (6) [see also (11)] we obtain

$$
p_{\varphi}=m r_{n}^{2} \dot{\varphi}=L_{n}=n \hbar .
$$

In effect (19) and (20) give the result:

$$
r=r_{n}=\frac{p_{\varphi}^{2}}{m e^{2}}=\frac{n^{2} \hbar^{2}}{m e^{2}}
$$

which is equivalent to a well-known formula for the radius $r_{n}$ of the $n$th Bohr orbit in the hydrogen atom; see e.g. [13].

\section{Application of the Formulae (7) and (10) in Calculating the Left-Hand Side of (8)}

Because of (7) and (10) the left-hand side of (8) can be transformed as follows:

$$
\frac{2 \bar{E}_{\text {kin }}}{v}= \pm \frac{4 \pi E_{\text {kin }}}{\dot{q}}= \pm \frac{2 \pi p \dot{q}}{\dot{q}}= \pm 2 \pi p_{\varphi}= \pm 2 \pi m r_{n}^{2} \dot{\varphi}= \pm 2 \pi n \hbar .
$$

The last steps in (22) are dictated by (6) and (20).

A constant value of the angular velocity $\dot{\varphi}$ can be represented by the ratio:

$$
\dot{\varphi}=\frac{2 \pi}{\Delta t}
$$

where $\Delta t$ is the time period of the electron circulation along the orbit. A substitution of (21) and (23) into (22) gives:

$$
m\left(\frac{n^{2} \hbar^{2}}{m e^{2}}\right)^{2} \frac{2 \pi}{\Delta t}=n \hbar .
$$

Therefore

$$
\Delta t=\frac{(n \hbar)^{3} 2 \pi}{m e^{4}}=\frac{2 \pi n^{3} \hbar^{3}}{m e^{4}} .
$$

This is a result obtained earlier (see [9] [10] [11]) on the basis of the quantum formulation of the Joule-Lenz law applied to the hydrogen atom. In fact, the time intervals deduced from the Joule-Lenz law were widely applied to the emission spectrum of the hydrogen atom; see [14] [15] [16] and [17]. 


\section{Mechanical Parameters of the Electron in the Hydrogen Atom Applied in Calculating the Time Intervals of Electron Transitions}

In a recent paper, we found [12] that the time intervals similar to that in (25) can be obtained when the mechanical parameters of the electron motion in the hydrogen are taken into account. For example, let the change of the electron position on an orbit between two quantum states

$$
n+1, n
$$

characterized by the change of the orbit length

$$
\begin{aligned}
2 \pi \Delta r_{n} & =2 \pi\left(r_{n+1}-r_{n}\right)=2 \pi\left[\frac{(n+1)^{2} \hbar^{2}}{m e^{2}}-\frac{n^{2} \hbar^{2}}{m e^{2}}\right] \\
& =2 \pi(2 n+1) \frac{\hbar^{2}}{m e^{2}} \approx 4 \pi n \frac{\hbar^{2}}{m e^{2}}
\end{aligned}
$$

is examined together with the change of the electron velocity

$$
\left|\Delta v_{n}\right|=\frac{e^{2}}{\hbar}\left|\frac{1}{n+1}-\frac{1}{n}\right|=\frac{e^{2}}{\hbar} \frac{n+1-n}{(n+1) n} \approx \frac{e^{2}}{\hbar n^{2}}
$$

concerning the same orbits. For if we put

$$
\Delta t=\frac{2 \pi \Delta r_{n}}{\left|\Delta v_{n}\right|},
$$

we obtain from (27), (28) and (29)

$$
\Delta t=\frac{2 \pi \Delta r_{n}}{\left|\Delta v_{n}\right|} \approx 4 \pi n \frac{\hbar^{2}}{m e^{2}} \frac{\hbar n^{2}}{e^{2}}=4 \pi \frac{(n \hbar)^{3}}{m e^{4}} \approx 3 \times 10^{-16} n^{3} \mathrm{sec}
$$

which is a much similar result to that calculated in (25): a difference between (25) and (30) is given by a factor of 2. A printing error done in calculating $\Delta t$ entering (30) in Ref. [12], Equation (19), has been removed.

\section{Landau Levels in the Electron Gas and Their Semiclassical Properties}

A well-known treatment of the Landau energy levels in the electron gas has been obtained with the aid of the free-electron oscillations examined on the basis of the quantum theory [18] [19] [20]. The energy levels due to the action of a constant magnetic field $B$ on the gas expressed in cylindrical coordinates $r, \varphi, z$, are [19]

$$
E=\left(n+j+\frac{1}{2}\right) \frac{e B}{c m} \hbar+\frac{\hbar^{2} k_{z}^{2}}{2 m}
$$

and their important property is that-because of integer $n$ and $j$-they are separated by a constant energy amount in a plane normal to a constant magnetic field $B$ ( $k_{z}=$ const $)$ : 


$$
\Delta E=\frac{e B}{c m} \hbar=\omega \hbar .
$$

The electron wave functions are

$$
\psi=R(r) \exp (i j \varphi) \exp \left(i k_{z} z\right)
$$

and $\omega$ in (32) is a circular electron oscillation frequency in the field $B$. The time period of the oscillation is $T$.

The constant frequency entering (32)

$$
\omega=\frac{e B}{c m}
$$

is coupled with $T$ by the formula

$$
\omega=2 \pi v=\frac{2 \pi}{T}
$$

see [18] [19] [20]. Because of (3) and (4) we obtain

$$
\Delta t=\frac{h}{\Delta E}=\frac{h}{\hbar \omega}=\frac{2 \pi}{\omega}=T
$$

which formally is a result much similar to that calculated earlier for $\Delta t$ in the hydrogen atom; see (25).

A semiclassical treatment of the electron motion in the magnetic field has been done in [19]. One of results concerns the radius of the orbit occupied by an electron circulating in the magnetic field. In case the electrons in the magnetic field are considered in cylindrical coordinates, the radial part of the wave function

$$
R(r)
$$

[see (33)] satisfies the Schrödinger equation by the formula [19]

$$
-\frac{\hbar^{2}}{2 m}\left(\frac{\mathrm{d}^{2} R}{\mathrm{~d} r^{2}}+\frac{1}{r} \frac{\mathrm{d} R}{\mathrm{~d} r}-\frac{j^{2}}{R^{2}}\right)+\frac{e^{2} B^{2}}{8 c^{2} m} r^{2} R=\left(E-\frac{j \hbar e B}{2 m c}-\frac{k_{z}^{2} \hbar^{2}}{2 m}\right) R
$$

where $j$ and $k_{z}$ are quantum numbers which refer to the $\varphi$-dependent and $z$-dependent part of the cylindrical wave function, respectively.

In general the function $R(r)$ in (37) has $n$ nodes. An equivalent of the classical orbit of an electron is that for which

$$
n=0 .
$$

In effect of (39) we have [19]

$$
R(r)=r^{j} \exp \left[-(\pi e B / 2 h c) r^{2}\right] .
$$

Consequently to (39) the planar part of the electron energy is reduced to the dependence on a single quantum number $j$ :

$$
E=E_{j}=\left(j+\frac{1}{2}\right) \hbar \frac{e B}{c m}
$$

giving the same energy interval as that presented in (32) on condition we put

$$
\Delta j=1 .
$$


The wave function (40) has a maximum at certain value value of $r$ and can be expanded approximately as a Gaussian function about that maximum. This treatment gives for the orbit radius $r$ the formula [19] [21] [22]

$$
\pi r^{2}=\frac{j h c}{e B}
$$

So consequently

$$
r=\frac{1}{\pi^{1 / 2}}\left(\frac{j h c}{e B}\right)^{1 / 2} .
$$

\section{Confirmation of the Results Attained in Sec. 6 by the Mechanical Properties of the Electron Motion in the Magnetic Field}

A semiclassical treatment of the free-electron motion in the magnetic field done in Sec. 6 gives the radius (44) of the electron orbit due to the applied field. We demonstrate now that the formula (36) for the transition time between Landau levels can be obtained with the aid of a simple mechanical reasoning.

The change of $r_{j}$ is connected with the change of $j$; see (44). Since the orbit length is

$$
l_{j}=2 \pi r_{j}
$$

for the quantum transition

$$
j+1 \rightarrow j
$$

We obtain the change

$$
\Delta l_{j}=2 \pi \Delta r_{j}=2 \pi\left(r_{j+1}-r_{j}\right)=\frac{2 \pi}{\pi^{1 / 2}}\left(\frac{h c}{e B}\right)^{1 / 2}\left[(j+1)^{1 / 2}-j^{1 / 2}\right] .
$$

The electron velocity $v_{j}$ on the orbit $j$ is given by the relation

$$
E_{j}=\frac{m v_{j}^{2}}{2}
$$

So

$$
v_{j}=\left(\frac{2 E_{j}}{m}\right)^{1 / 2} .
$$

In effect because of (49) we obtain the velocity change

$$
\begin{aligned}
\Delta v_{j} & =\left(\frac{2}{m}\right)^{1 / 2}\left(E_{j+1}^{1 / 2}-E_{j}^{1 / 2}\right) \\
& =\left(\frac{2}{m}\right)^{1 / 2}\left(\frac{\hbar e B}{c m}\right)^{1 / 2}\left[\left(j+1+\frac{1}{2}\right)^{1 / 2}-\left(j+\frac{1}{2}\right)^{1 / 2}\right] .
\end{aligned}
$$

But for large $j$ we have

$$
j \approx j+\frac{1}{2} .
$$

So expression (50) becomes approximately equal to 


$$
\Delta v_{j} \cong\left(\frac{2}{m}\right)^{1 / 2}\left(\frac{\hbar e B}{c m}\right)^{1 / 2}\left[(j+1)^{1 / 2}-j^{1 / 2}\right] .
$$

From the mechanics we have

$$
\Delta v_{j}=\frac{\Delta l_{j}}{\Delta t}
$$

so from (53):

$$
\begin{aligned}
\Delta t & =\frac{\Delta l_{j}}{\Delta v_{j}} \cong \frac{2 \pi^{1 / 2}\left(\frac{h c}{e B}\right)^{1 / 2}\left[(j+1)^{1 / 2}-j^{1 / 2}\right]}{\left(\frac{2}{m}\right)^{1 / 2}\left(\frac{\hbar e B}{c m}\right)^{1 / 2}\left[(j+1)^{1 / 2}-j^{1 / 2}\right]} \\
& =\frac{(2 \pi)^{3 / 2}}{2^{1 / 2} \pi^{1 / 2}} \frac{c m}{e B}=\frac{2 \pi}{\omega} .
\end{aligned}
$$

This is precisely the result for $\Delta t$ obtained in (36).

\section{Summary}

In the first step, an analysis of the Ehrenfest invariant demonstrated that the time interval necessary for the energy emission from level $n+1$ to level $n$ in the hydrogen atom can be deduced from the electron orbital momentum on level $n$. This interval is found to be precisely equal to the time dictated by the Joule-Lenz law for the electron transition from $n+1$ to $n$ [9].

In the next step, the time interval due to the Joule-Lenz law connected with the emission of energy between two neighbouring quantum levels is examined with the aid of the mechanical properties possessed by the electron. In the case of the hydrogen atom the time interval calculated in this way occurs to be twice as large as the interval given by the Joule-Lenz formula; see Sec. 5. Another use of the mechanical parameters is done for calculating the transition time between two neighbouring Landau levels in the electron gas. In this case, when the large quantum numbers are considered for the levels, the time intervals obtained from the Joule-Lenz approach and those with the use of the mechanical parameters become the same (see Sec. 7 and [9]).

\section{Conflicts of Interest}

The authors declare no conflicts of interest regarding the publication of this paper.

\section{References}

[1] Planck, M. (1932) Einführung in die Theorie der Wärme. S. Hirzel, Leipzig.

[2] Einstein A. (1917) The Quantum Theory of Radiation. Physikalische Zeitschrift, 18, 121.

[3] Van der Waerden B. L. (1967) Sources of Quantum Mechanics. Dover, New York.

[4] Schiff L. I. (1968) Quantum Mechanics. 3rd Edition, McGraw-Hill, New York.

[5] Slater J. C. (1960) Quantum Theory of the Atomic Structure. Volume 1, McGraw- 
Hill, New York.

[6] Ehrenfest, P. (1916) Adiabatische Invarianten und Quantentheorie. Annalen der Physik, 51, 327-352. https://doi.org/10.1002/andp.19163561905

[7] Ehrenfest, P. (1917) Adiabatic Invariants and the Theory of Quanta. Philosophical Magazine, 33, 500-513. https://doi.org/10.1080/14786440608635664

[8] Lass, H. (1950) Vector and Tensor Analysis. McGraw-Hill, New York. https://doi.org/10.1119/1.1932684

[9] Olszewski, S. (2015) Non-Probabilistic Approach to the Time of Energy Emission in Small Quantum Systems. Journal of Modern Physics, 6, 1277-1288. https://doi.org/10.4236/jmp.2015.69133

[10] Olszewski, S. (2016) Quantum Aspects of the Joule-Lenz Law. Journal of Modern Physics, 7, 162-174. https://doi.org/10.4236/jmp.2016.71018

[11] Olszewski, S. (2016) The Bohr Model of the Hydrogen Atom Revisited. Reviews in Theoretical Science, 4, 336-352. https://doi.org/10.1166/rits.2016.1066

[12] Olszewski, S. (2019) Time Intervals of the Electron Transitions between the Energy States in the Hydrogen Atom Calculated in a Non-Probabilistic Way. Journal of Modern Physics, 10, 1522-1531. https://doi.org/10.4236/jmp.2019.1013101

[13] Sommerfeld A. (1931) Atombau und Spektrallinien. 5th Edition, Vieweg, Braunschweig.

[14] Olszewski, S. (2016) Emission Intensity in the Hydrogen Atom Calculated from a Non-Probabilistic Approach to the Electron Transitions. Journal of Modern Physics, 7, 827-851. https://doi.org/10.4236/jmp.2016.78076

[15] Olszewski, S. (2016) Semiclassical and Quantum-Mechanical Formalism Applied in Calculating the Emission Intensity of the Atomic Hydrogen. Journal of Modern Physics, 7, 2314. https://doi.org/10.4236/jmp.2016.79091

[16] Olszewski, S. (2017) Time Intervals of the Electron Transitions and Intensity Spectrum of the Hydrogen Atom. Journal of Computational and Theoretical Nanoscience, 14, 4086-4099. https://doi.org/10.1166/jctn.2017.6791

[17] Condon, E.U. and Shortley, G.M. (1970) The Theory of Atomic Spectra. University Press, Cambridge (UK).

[18] Landau, L.D. (1930) Diamagnetismus der Metalle. Zeitschrift für Physik, 64, 629-637. https://doi.org/10.1007/BF01397213

[19] Slater, J.C. (1967) Quantum Theory of Molecules and Solids. Volume 3, McGrawHill, New York.

[20] Kittel, C. (1987) Quantum Theory of Solids. 2nd Edition, Wiley, New York.

[21] Fock, V. (1928) Bemerkung zur Quantelung des harmonischen Oszillators im Magnetfeld. Zeitschrift für Physik, 48, 446-448. https://doi.org/10.1007/BF01390750

[22] Dingle, R.B. (1952) Some Magnetic Properties of Metals. I. General Introduction, and Properties of Large Systems of Electrons. Proceedings of the Royal Society (London), A211, 500-525. https://doi.org/10.1098/rspa.1952.0055 